УДК 622.411

\title{
АНАЛИТИЧЕСКИЙ ПОДХОД К ОЦЕНКЕ ЭФФЕКТА ВОДЯНОЙ ПРОБКИ В ВЕНТИЛЯЦИОННЫХ СТВОЛАХ ШАХТ
}

\author{
Шалимов Андрей Владимирович', \\ shalimovav@mail.ru
}

\section{Казаков Борис Петрович1,} aero_kaz@mail.ru

\author{
Зайцев Артем Вячеславович1, \\ aerolog.artem@gmail.com \\ 1 Горный институт УрО РАН, \\ Россия, 614007, г. Пермь, ул. Сибирская, 78а.
}

\begin{abstract}
Актуальность исследования обусловлена необходимостью снижения дополнительной нагрузки на главные вентиляторные установки, связанной с возникновением обратной тяги при капеже в вентиляционных стволах шахт и рудников. Наиболее остро проблема нормализации работы вентилятора возникает в случае возможного перехода его в режим неустойчивой работы, что сопряжено с необходимостью не только энергосбережения, но и предотвращения его аварийного останова.

Цель: выяснение причин и механизма возникновения эфффекта «водяной пробки» в вентиляционных стволах с получением качественных и количественных оценок величины обратной тяги в зависимости от скорости движения воздуха, әлубины и интенсивности источника выделения влаги.

Объекты: вентиляционные стволы шахт.

Методы: аналитическое моделирование процессов конденсации влаги и движения капель в восходящем воздушном потоке; сравнительный анализ экспериментальных и модельных данных.

Результаты. Проведён анализ экспериментальных данных по образованию и движению капельной влаги в вентиляционных стволах различных рудников. Отмечено, что эфрфект водяной пробки наблюдается в ограниченном диапазоне скоростей движения воздуха от 7 до 12 м/с, но в некоторых случаях эфрфект отсутствует, даже несмотря на наличие густого тумана и обильной конденсации влаги на поверхности крепи и армировки ствола. Рассмотрены три возможных сиенария возникновения эффректа: накопление большого количества зависающих в воздухе капель определённого размера; падение капель крупных размеров по всей глубине ствола с источником выделения влаги в его верхней части и разнонаправленное движение капель в зависимости от их размера с источником влаговыделения, расположенным на произвольной глубине. Установлено, что первые две модели не позволяют получить количественную оценку величины эффректа ввиду неопределённости фрракционного состава капель, критический размер зависания которых увеличивает обратную тягу до бесконечности. Этот недостаток устранён в третьей модели введением функции распределения капель по размерам, что дало возможность получить количественные результаты. Доказано, что выделение влаги из воздуха не может являться причиной возникновения эфрфекта «водяной пробки», а причиной являются водопритоки в ствол с интенсивностью, на порядок превышающей конденсацию. По результатам численного моделирования показано, что максимальная обратная тяга возникает при проникновении в ствол поверхностных грунтовых вод.
\end{abstract}

\section{Ключевые слова:}

Рудничная вентиляция, конденсация влаги, гидростатическое охлаждение, водопритоки, депрессия, аэродинамическое сопротивление, естественная тяга, фракционный состав.

\section{Введение}

Естественная тяга в рудниках возникает в результате действия трёх факторов - ветра, разности плотностей воздуха и капежа в стволах, влияние которых на проветривание может быть сильным или слабым в зависимости от условий. Воздействие естественной тяги может быть положительным и улучшать вентиляцию или отрицательным и препятствовать движению воздуха через рудник под действием депрессии главной вентиляторной установки, в случае же её отключения конвективное движение воздуха становится неустойчивым по направлению и интенсивности [1-3] Влияние ветра на проветривание наблюдается в карьерах [4], тоннелях [5] и подземных горнодобывающих предприятиях с горизонтальными стволами - штольнями. Основной, широко распространённой и хорошо изученной [6] причиной возникновения естественной тяги в рудниках с вертикальными стволами является разность плотностей воздуха, обусловленная главным образом разными значениями его температуры в воздухоподающих и вентиляционных стволах. Явление капежа в шахтных стволах изучено в значительно меньшей степени [7], встречается реже и, как правило, оказывает более слабое влияние на проветривание. Однако в некоторых случаях чрезмерное скопление влаги в вентиляционном стволе приводит к аварийной ситуации, связанной с возникновением сильной обратной тяги, результатом чего является выход вентилятора из-за перегрузки на режим неустойчивого проветривания в область помпажа [8], что недопустимо правилами безопасности.

В условиях высокой влажности восходящего воздушного потока может возникать такое опасное явление, как Бланкет-эффект, или эффект «водяной пробки», суть которого связывается многими авторами с водопритоками и конденсацией избыточной влаги из 
воздуха с её постепенным накоплением в стволе [9-13]. На практике это выражается в появлении в вентиляционных стволах густого тумана, снижении расхода воздуха, увеличении депрессии вентилятора и последующем его аварийном отключении. В [10, 14] указывается, что обратная тяга возникает в вентиляционных стволах шахт в интервале скоростей движения воздуха 7-12 м/с. По данным [10] Бланкет-эффект впервые был обнаружен в 1950-х гг. в связи с ситуацией в вентиляционном стволе одной из шахт в г. Вал-Риф в ЮАР, которая проветривалась всасывающим способом с помощью главной вентиляторной установки, состоящей из двух вентиляторов, работающих в параллели. Вентиляционный ствол шахты имел избыточную влажность из-за наличия трещин в крепи ствола с обильными водопритоками из окружающих водоносных горизонтов вблизи дневной поверхности. В стволе была предусмотрена система от- качки воды на поверхность по трубопроводу, и когда трубопровод или насос оказывались неисправными, вода лилась в ствол, в результате чего образовывался сильный туман и перепад давлений на вентиляторах значительно возрастал. Проблема решалась выключением одного из вентиляторов, в результате чего скорость воздуха в стволе падала до $7 \mathrm{M} / \mathrm{c}$, туман в стволе пропадал, и, как следствие, аэродинамическое сопротивление ствола уменьшалось. Один вентилятор продолжал работать до тех пор, пока не была устранена неисправность в системе откачки воды на поверхность, после чего включался второй. В работе [9] также описываются случаи, когда поверхностные вентиляторные установки в условиях высокой влажности воздуха в вентиляционных восстающих выработках переходили в нестабильный режим работы, который приводил к усталостным повреждениям конструкций.

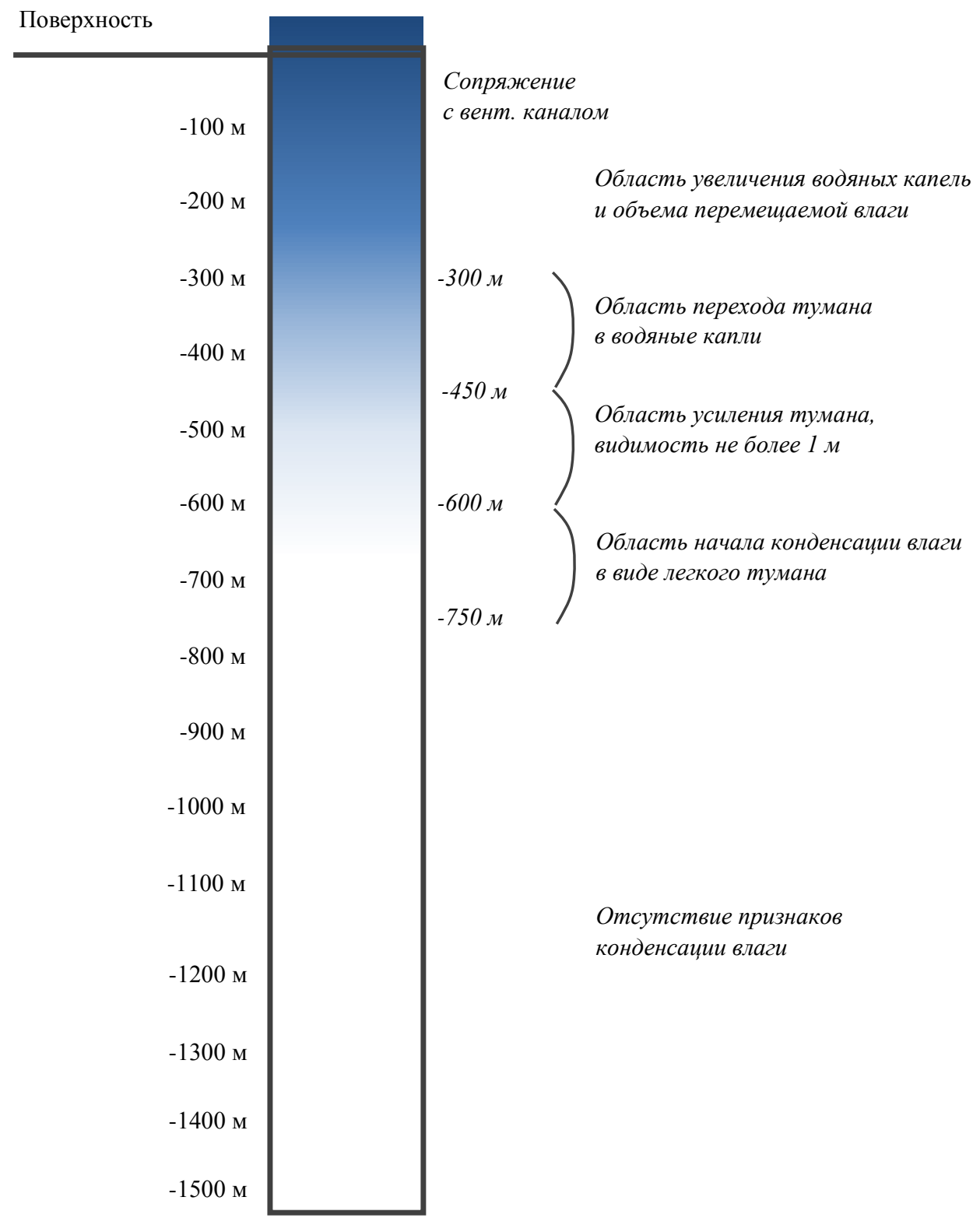

Puc. 1. Процесс конденсаиии влаги в стволе ВC-7

Fig. 1. Moisture condensation in the VS-7 mine shaft 
Аналогичная ситуация имела место в вентиляционном стволе ВС-6 рудника Таймырский ОАО «ГМК "Норильский никель"». В 2011-2013 гг. сотрудники Горного института УрО РАН проводили исследования системы вентиляции этого рудника, по результатам которых было установлено, что во время проведения замеров расход главной вентиляторной установки ВЦД-47 «Север» снижался, а ее депрессия возрастала. Были проведены также детальные обследования вентиляционных стволов ВС-3, BC-4, BC-6 и $\mathrm{BC}-7$ рудников Таймырский и Октябрьский ОАО «ГМК "Норильский никель"». В ходе обследования осуществлялся спуск-подъем на бадье по стволу от надшахтного здания до основных подземных горизонтов, и было установлено, что при движении воздуха вверх в какой-то момент в стволе начинает появляться туман. По мере продвижения вверх туман становился гуще, начинал конденсироваться на армировке ствола, наиболее густой туман наблюдался вверху ствола до сопряжения с вентиляционным каналом. Наряду с туманом были отмечены также процессы падения крупных капель влаги вниз и движения мелкодисперсной капельной влаги вверх вместе с воздушным потоком с последующим выносом в вентиляционный канал. На рис. 1 представлено схематичное отображение участков ствола ВС-7 с разделением по участкам конденсации влаги.

Движущийся по стволу ВС-7 воздух на сопряжениях с горизонтами имеет высокую влажность и температуру. В процессе движения происходит постепенное охлаждение воздуха и конденсация содержащейся в нем влаги. Начало процесса конденсации влаги происходит в интервале отметок от -750 до 600 м с образованием легкой туманности. По мере движения воздуха вверх по стволу и дальнейшего охлаждения туман становится гуще, и в интервале глубин от -450 до -300 м происходит образование водяных капель. Далее каплеобразование усиливается за счет слияния более мелких капель воды, часть влаги стекает по стенкам крепи в зумпф, а часть поднимается восходящим потоком воздуха и выносится в вентиляционный канал. Но следует отметить, что в стволе ВС-7, несмотря на признаки обильной конденсации влаги, эффекта «водяной пробки» обнаружено не было, что указывает на необходимость изучения эффекта не только с качественной, но и количественной стороны с рассмотрением всевозможных сценариев эффекта и оценкой влияния всех факторов его возникновения.

Ввиду недостаточного количества экспериментальной информации по условиям возникновения этого малоизученного эффекта наиболее целесообразным является разработка комплекса оценочных аналитических моделей, которые позволили бы в упрощённой форме смоделировать исследуемый процесс и выявить основные факторы, определяющие величину обратной тяги. В работе [15] представлена одна из таких моделей зависания и накопления в стволе капель при критической скорости движения воздуха $\sim 9$ м/с без учёта зависимости скорости движения капель от их размеров. Представленные ниже модели являются продолжением этой работы по исследованию эффекта водяной пробки с учётом фракционного состава капель.

\section{Математическое моделирование Бланкет-эффекта в вентиляционном стволе}

Сначала рассматривается наиболее очевидный сценарий возникновения дополнительной нагрузки на вентилятор. Влажный воздух поднимается по вентиляционному стволу, охлаждается, достигая точки росы. Влага выпадает в виде капель, которые в зависимости от соотношения их размеров и скорости движения воздуха падают, поднимаются или зависают на месте. Фракционный состав будет разный, но интерес представляет размер тех капель, которые будут оставаться на месте, поскольку именно они будут накапливаться и, как предполагаемый результат, создавать воздушную пробку, препятствующую прохождению воздушного потока. Оценить размер «зависших» капель можно, приравняв силу их лобового сопротивления воздушному потоку к силе тяжести [16]

$$
k \frac{\rho_{a} \omega^{2}}{2} f=g \rho_{w} V,
$$

$\rho_{a}$ и $\rho_{w}-$ плотности воздуха и воды, кг $/ \mathrm{M}^{3} ; g$ - ускорение силы тяжести, м/ $\mathrm{c}^{2} ; \omega-$ скорость движения воздуха, м/с; $V=\frac{4}{3} \pi r^{3}$ - объём капли, м $; f=\pi r^{2}$ - сечение капли по воздуху, м $^{2} ; r$ - радиус капли, м; $k=0,4-$ коэффициент аэродинамического сопротивления шара при числе $R e=r \omega / v \sim 1000$, безр.; $v$ - кинематическая вязкость воздуха, ${ }^{2}$,с.

При подстановке в (1) $r$ выражается через $\omega$

$$
r=\frac{1}{5 g} \frac{\rho_{a}}{\rho_{w}} \omega^{2} .
$$

В диапазоне скоростей движения воздуха от 7 до $12 \mathrm{м} / \mathrm{c}$ критический размер $2 r$ «зависающих» частиц изменяется от 2 до 6 мм, что согласуется с результатами метеорологических наблюдений за скоростью падения капель дождя в зависимости от их размеров [17].

Зная количество висящей в воздухе влаги в виде таких капель, можно записать баланс направленных в разные стороны общих сил тяжести и депрессии

$$
M g=S \Delta P,
$$

$M=N \rho_{w} 4 \pi r^{3} / 3-$ общая масса «зависшей» в стволе влаги, кг; $N$ - общее количество капель; $S$ - сечение ствола, ${ }^{2} ; \Delta P=\Delta R(\omega S)^{2}$ - часть депрессии вентилятора, идущая на поддержание на весу всей массы капель $M$, Па; $\Delta R$ - аэродинамическое сопротивление капельной взвеси, кг/м ${ }^{7}$. Значит

$$
\Delta R=\frac{M g}{\omega^{2} S^{3}} .
$$

Оценить $N$ можно исходя из предположения стационарности Бланкет-эффекта. Если предположить, что влага в стволе не накапливается, а постоянно обновляется за счёт конденсации из воздуха, то количество конденсируемой на «пробочном» участке влаги с 
размером капель $r$ должно быть равно количеству уходящей с участка влаги вниз или вверх с другими размерами капель. Значит, число образующихся для поддержания постоянного количества капель равно избыточному влагосодержанию воздуха $\Delta \eta, \quad \kappa г / \mathrm{m}^{3}$, при подъёме по стволу, отнесённому к массе одной капли

$$
\begin{gathered}
m_{0}=\frac{4}{3} \pi \rho_{w} r^{3}, \text { кг, } \\
\frac{N}{S H} \sim \frac{\Delta \eta}{m_{0}},
\end{gathered}
$$

$H$ - высота капельной пробки, м. При остывании воз-

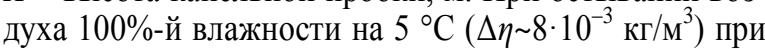
подъёме с глубины в 1 км для капель размером $2 r=2$ мм их стационарная концентрация составит примерно 2000 шт/м ${ }^{3}$. При $H=500$ м и $S=60$ м $^{2}$ $M=S H \Delta \eta$ составит около 250 кг независимо от размера капель, что соответствует потере депрессии в 40 Па, а это на порядок меньше наблюдаемых значений. Таким образом, предположение (5) количественно эффект не объясняет, и, если сценарий зависания капель верен, должно происходить накопление влаги в стволе до концентраций, в 10 превышающих оценённые по предположению (5).

Возможно другое объяснение механизма возникновения дополнительного аэродинамического сопротивления, основанное не на накоплении и зависании капель в стволе. Избыточная влага в результате гидростатического охлаждения воздуха в верхней части ствола конденсируется, коагулирует в крупные капли и падает вниз. По всей глубине ствола идёт дождь, вызывающий за счёт трения капельную естественную тягу, направленную против депрессии вентилятора.

Зависимость (2) меняется с учётом того, что капли уже не висят в воздухе на одной высотной отметке, а падают вниз

$$
r=\frac{1}{5 g} \frac{\rho_{a}}{\rho_{w}}\left(\omega+\omega^{\prime}\right)^{2},
$$

$\omega$ - скорость движения воздуха относительно ствола, м/с; $\omega^{\prime}-$ скорость движения капли относительно ствола, м/с.

Падающие вниз капли из-за сопротивления воздуха быстро набирают предельную скорость и двигаются вниз без ускорения. Поэтому зависимость (3) остаётся прежней в предположении стационарности процессов конденсации и выпадения влаги внизу ствола. Предполагается, что образующиеся мелкие капли быстро коагулируют, становятся тяжёлыми и не выносятся исходящим потоком, а только падают вниз

$$
M g=S \Delta P,
$$

$M=N m_{0}$ - общая масса находящейся в стволе в каждый момент времени капельной влаги, кг; $N$ - общее количество капель в стволе в каждый момент времени $S$ - сечение ствола, м $^{2} ; \Delta P=\Delta R(\omega S)^{2}-$ часть депрессии вентилятора, идущая на преодоление сопротивления движению воздуха падающих капель, Па; $\Delta R$ - аэродинамическое сопротивление, в отличие от (4), не висящей в стволе, а падающей капельной взвеси, кг $/ \mathrm{M}^{7}$.
Стационарность процесса означает, что количество выпадающей в зумпф влаги должно быть равно количеству влаги, образующейся вверху в результате конденсации

$$
\omega^{\prime} n=\frac{\omega \Delta \eta}{m_{0}},
$$

где $n$ - концентрация капель в воздухе, $\mathrm{m}^{-3}$, может быть выражена через $\Delta P$ из (7). В результате (8) и (6) образуют систему уравнений для нахождения $\omega^{\prime}$ и $r$

$$
\left\{\begin{array}{c}
\frac{\omega^{\prime} \Delta P}{g H}=\omega \Delta \eta ; \\
\left(\omega^{\prime}+\omega\right)^{2}=5 r g \frac{\rho_{w}}{\rho_{a}} .
\end{array}\right.
$$

После исключения $\omega^{\prime}$ из (9)

$$
r=\frac{1}{5 g} \frac{\rho_{a}}{\rho_{w}} \omega^{2}\left(1+\frac{g H \Delta \eta}{\Delta P}\right)^{2} .
$$

Оценка $r$ для $\omega=10 \mathrm{~m} / \mathrm{c}, H=1 \mathrm{\kappa м}, \Delta \eta=10$ г $/ \mathrm{M}^{3}$ (соответствует гидростатическому охлаждению воздуха $100 \%$-й влажности на $\left.5^{\circ} \mathrm{C}[18]\right)$ и $\Delta P=500$ Па даёт размер $r=3$ мм, соответствующий каплям крупного дождя [17]. Таким образом, второй вариант объяснения Бланкет-эффекта предпочтительнее, т. к. проще и не содержит неизвестного параметра - количества скопившейся висячей влаги. Кроме того, водопритоки в ствол могут быть учтены простой добавкой к $\Delta \eta$ : $\Delta \eta+w /(\omega S)$, где $w-$ скорость притока воды в ствол (кг/c). Подлежащим определению параметром является $\Delta P$, поэтому (10) лучше переписать в виде

$$
\Delta P=\frac{g H \Delta \eta}{\sqrt{\frac{\rho_{w}}{\rho_{a}} \frac{5 g r}{\omega^{2}}}-1} .
$$

На первый взгляд зависимость (11) выглядит нелогично, т. к. при определённом соотношении параметров знаменатель становится нулевым или отрицательным. Но если вспомнить, что в модели принято, что капли падают вниз, а это возможно лишь при соблюдении неравенства $r>\frac{1}{5 g} \frac{\rho_{a}}{\rho_{w}} \omega^{2}$, то противоречия нет.

Однако информационная польза результата (11) второй модели невелика, т. к. искомый параметр $\Delta P$ может быть получен сколь угодно большим в зависимости от размера капель $r$, являющегося неопределённой величиной, и при равенстве (2) дающий $\Delta P \rightarrow \infty$.

Этот недостаток аналитического моделирования можно устранить, если учесть, что капли образуются всех возможных размеров, не превышающих максимальный устойчивый [19]. Следует учесть также, что выделение влаги может происходить не только в верхней части ствола. По-прежнему принимается упрощение сосредоточенного источника влаги, только теперь он может находиться на любой высотной отметке $h, \mathrm{M}$, отсчитываемой от поверхности (от 0 до $H$ ). Считается 
также, что концентрация капель во всём объёме воздуха ствола в стационарном режиме распределена равномерно по массе, т. е. на каждую фракцию приходится одинаковая масса влаги по соображениям хаотичности образования, распада и слияния капель:

$$
n(r) r^{3}=A=\mathrm{const}
$$

$n(r)$ - функция распределения концентрации капель по размерам, $\mathrm{M}^{-4}$.

Поскольку источник влаги находится теперь не в верхней точке ствола, а на глубине $h$, то в создании дополнительного аэродинамического сопротивления ствола будут участвовать как падающие капли, так и восходящие, и падение давления $\Delta P$ из (3) будет иметь вид

$$
\Delta P=\frac{4}{3} \pi \rho_{w} g\left[(H-h) \int_{r_{\min }}^{r_{\max }} n(r) r^{3} d r+h \int_{0}^{r_{\min }} n(r) r^{3} d r\right],
$$

здесь

$$
r_{\min }=\frac{1}{5 g} \frac{\rho_{a}}{\rho_{w}} \omega^{2}
$$

- критический радиус капли для такой скорости движения воздуха $\omega$, при которой она зависает относительно ствола (падение сменяется подъёмом); $r_{\max } \geq r_{\min }$ - максимальный радиус устойчивости капли, выше которого капля разваливается потоком воздуха, м (по метеорологическим данным $r_{\max }=3 \mathrm{Mм}$ ).

При «включении» источника выделения влаги процесс сначала имеет нестационарный характер - концентрация влаги в воздухе для всех размеров капель растёт. Но с ростом концентрации интенсивности падения крупных и выноса мелких капель увеличиваются, и наступает момент, когда общий вынос влаги из ствола становится равным выделению влаги $\Delta \eta$. С этого момента процесс влагообмена становится стационарным - сколько влаги выделяется, столько и выносится

$$
\omega \Delta \eta=\frac{4}{3} \pi \rho_{w} \int_{0}^{r_{\max }}\left|\sqrt{\frac{\rho_{w}}{\rho_{a}} 5 g r}-\omega\right| n(r) r^{3} d r .
$$

Система уравнений (12), (13)-(15) достаточна для определения $\Delta P$ :

$$
\Delta P=\frac{\frac{2}{3} g H \Delta \eta\left[\left(1-\frac{h}{H}\right)\left(\frac{r_{\max }}{r_{\min }}-1\right)+\frac{h}{H}\right]}{\left(\frac{r_{\max }}{r_{\min }}\right)^{3 / 2}-\frac{2}{3} \frac{r_{\max }}{r_{\min }}+1} .
$$

Определяющей зависимость $\Delta P$ от соотношений $h / H$ и $r_{\min } / r_{\max }$ является функция $f$, играющая роль безразмерного перепада давлений с единицей измерения $\frac{2}{3} g H \Delta \eta$,

$$
f\left(\frac{r_{\max }}{r_{\min }}, \frac{h}{H}\right)=\frac{\left(1-\frac{h}{H}\right)\left(\frac{r_{\text {max }}}{r_{\text {min }}}-1\right)+\frac{h}{H}}{\left(\frac{r_{\text {max }}}{r_{\text {min }}}\right)^{3 / 2}-\frac{2}{3} \frac{r_{\text {max }}}{r_{\text {min }}}+1},
$$

из вида которой можно заключить, что при $r_{\max }>2 r_{\min }$ (малая скорость движения воздуха) $\Delta P$ падает с увеличением $h$, а при $r_{\max }<2 r_{\min }$ (большая скорость движения воздуха) $\Delta P$ возрастает с увеличением $h$. Из анализа зависимости функции $f$ (17) от переменных $r_{\max } / r_{\min }$ и $h / H$ следует (рис. 2), что у неё есть максимальное значение $f_{\max }=0,75$ при $r_{\max } / r_{\min }=1$ и $h / H=1$, что соответствует выделению влаги в нижней точке ствола при максимальной скорости движения воздуха, обеспечивающей подъём всей образующейся влаги. При уменьшении глубины уровня влаговыделения $h$ $f_{\max }$ уменьшается с сохранением максимальной $\omega$ до значения $h / H \approx 0,6$, выше которого появляется экстремальный максимум с уменьшением значения $\omega$. С дальнейшим уменьшением $h$ величина экстремума растёт и достигает наибольшего значения $f \approx 0,48$ на поверхности $(h=0)$ при скорости движения воздуха, соответствующей $r_{\max } / r_{\min }=3,3$, которая всё же оказывается меньше краевого максимального значения функции $f_{\max }=0,75$ для источника выделения влаги в нижней части ствола. Оценка величины $\Delta P$ по максимуму даёт значение приблизительно 40 Па, что как минимум в 10 раз меньше ожидаемого в соответствии с опытными данными.

Из результатов проведённого анализа следует вывод, что требуемое для возникновения эффекта «водяной пробки» выделение влаги $\Delta \eta$ происходит не из воздуха путём конденсации [20], а из грунтовых или поверхностных вод, причём в количестве, превышающем на порядок конденсационное выделение. Поэтому избыточное влагосодержание воздуха $\Delta \eta\left(\right.$ кг $\left./ \mathrm{m}^{3}\right)$, приводящее к выделению влаги на глубине $h$, должно быть заменено на $w /(v S)$, где $w$ - скорость водопритоков на глубине $h$, кг/c; $v$ - скорость движения воздуха (в отличие от $\omega$ не ограниченная максимальным размером капли $\left.r_{\max }\right)$, м/c; $S$ - сечение ствола, м². Более информативной характеристикой в данном случае является зависимость $\Delta P$ от скорости движения воздуха, которую с учётом (14) также можно привести к безразмерной форме

$$
\bar{\omega}^{2}=\frac{r_{\min }}{r_{\max }}=\left(\frac{\omega}{\omega^{\prime}}\right)^{2},
$$

где $\omega^{\prime}=\sqrt{5 g \frac{\rho_{w}}{\rho_{a}} r_{\max }} \approx 12-$ единица измерения скорости движения воздуха, м/с.

В новых переменных (16) приобретает вид

$$
\Delta P=\frac{\frac{2}{3} g H w\left[\left(1-\frac{h}{H}\right)\left(\bar{\omega}^{-2}-1\right)+\frac{h}{H}\right]}{\omega^{\prime} S \bar{v}\left(\bar{\omega}^{-3}-\frac{2}{3} \bar{\omega}^{-2}+1\right)}
$$

где определяющей зависимость $\Delta P$ от $h / H$ и $\bar{\omega}$ является функция $F$ в качестве безразмерного перепада давлений с единицей измерения $\frac{2}{3} g H \frac{w}{\omega^{\prime} S}$ 


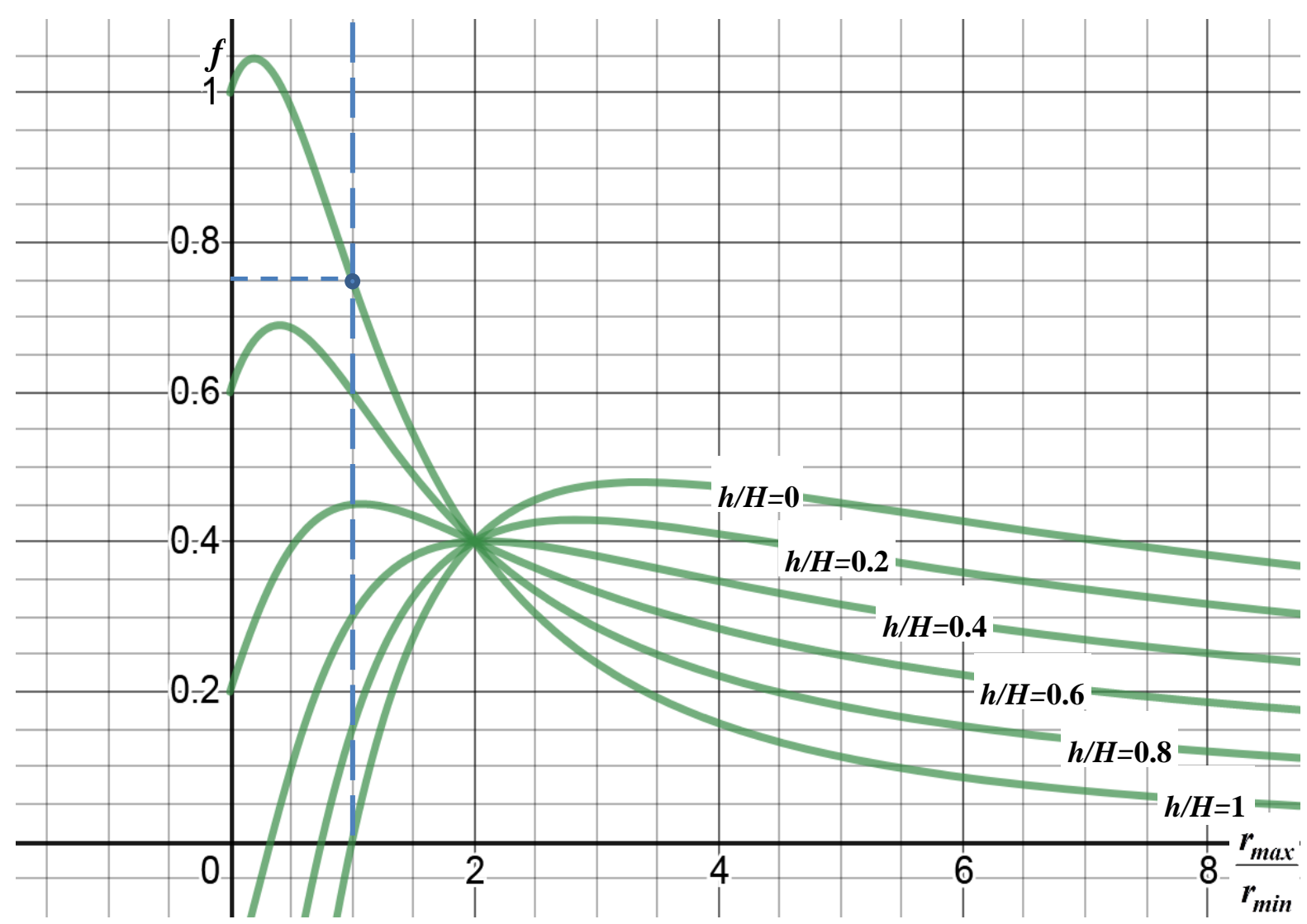

Pис. 2. Зависимость безразмерного перепада давлений $f$ от глубины места выделения влаги $0 \leq h / H \leq 1$ и минимального размера падающих вниз капель $1 \leq r_{\text {max }} / r_{\text {min }}<\infty$

Fig. 2. Dependence of the dimensionless pressure drop $f$ on the depth of the moisture release site $0 \leq h / H \leq 1$ and the minimum size of the drops falling down $1 \leq r_{\max } / r_{\min }<\infty$

$$
F\left(\bar{v}, \frac{h}{H}\right)=\frac{\left[\left(1-\frac{h}{H}\right)\left(\bar{\omega}^{-2}-1\right)+\frac{h}{H}\right]}{\bar{v}\left(\bar{\omega}^{-3}-\frac{2}{3} \bar{\omega}^{-2}+1\right)} .
$$

Здесь $\bar{\omega}=\left\{\begin{array}{c}\bar{v}, \quad 0 \leq \bar{v} \leq 1 \\ 1, \bar{v} \geq 1\end{array}\right.$, что означает ограничение

размера капель радиусом $r_{\max }$, выше которого их рост прекращается ввиду потери устойчивости, соответственно $\bar{\omega}$ достигает предельного значения 1 , а $\bar{v}$ ограничений не имеет. При дальнейшем росте скорости $\bar{v}>1$ выделяющаяся влага разбавляется нарастающим потоком воздуха, и $F$ уменьшается обратно пропорционально $\bar{v}$.

Из графиков функции $F$ (рис. 3 ) следует, что максимальное сопротивление воздушному потоку $(F=1,1)$ оказывают грунтовые воды, проникающие в ствол с поверхности, при скорости движения воздуха порядка 3 м/с. При более глубоком попадании воды в ствол максимум $F$ уменьшается до и возрастает после $h / H=0,5$ с увеличением $v$. При $h / H=0,4$ максимальный эффект достигается при $v=7 \mathrm{~m} / \mathrm{c}$, а в нижней части ствола при $v=12 \mathrm{M} / \mathrm{c}$ вся влага уносится вверх с $F=0,75$, что составляет примерно $70 \%$ от эффекта «водяной пробки», вызванного падением влаги с поверхности.

\section{Заключение}

Из результатов проведённого анализа следует вывод, что основной причиной возникновения Бланкетэффекта в вентиляционных стволах шахт являются поверхностные водопритоки в ствол, превышающие количественно на порядок конденсационное выделение влаги из воздуха при его подъёме с гидростатическим охлаждением. Несоответствие значения скорости движения воздуха $v=3 \mathrm{~m} / \mathrm{c}$ наблюдаемому на практике диапазону от 7 до 12 м/с объясняется, очевидно, грубостью модели сосредоточенного источника выделения влаги. В реальности вода стекает по крепи и расстрелам, и процесс отрыва капель от твёрдых поверхностей и попадания их в воздушный поток «растягивается» по глубине ствола, что увеличивает критическую скорость движения воздуха для достижения максимального эффекта. По экспериментальным данным величина обратной тяги порядка 500 Па, что согласно (18) соответствует водопритокам $w \sim 10$ кг/с и одномоментно падающей и восходящей в стволе влаги порядка 1 т на максимуме $F$. Снижение или увеличение скорости движения воздуха приводит к сокращению массы воды в воздухе путём падения части её вниз в первом случае или выноса вверх во втором.

Представленные в статье результаты моделирования эффекта «водяной пробки» в вентиляционных стволах могут быть использованы для профилактики 
этого негативного явления, а в случае его возникновения - для принятия мер по недопущению аварийного останова вентилятора и скорейшей нормализации его работы.
Исследование выполнено при финансовой поддержке Министерства науки и образования $Р \Phi$ в рамках соглашения по государственному заданию № 075-03-2021-374 от «29» декабря 2020 г.

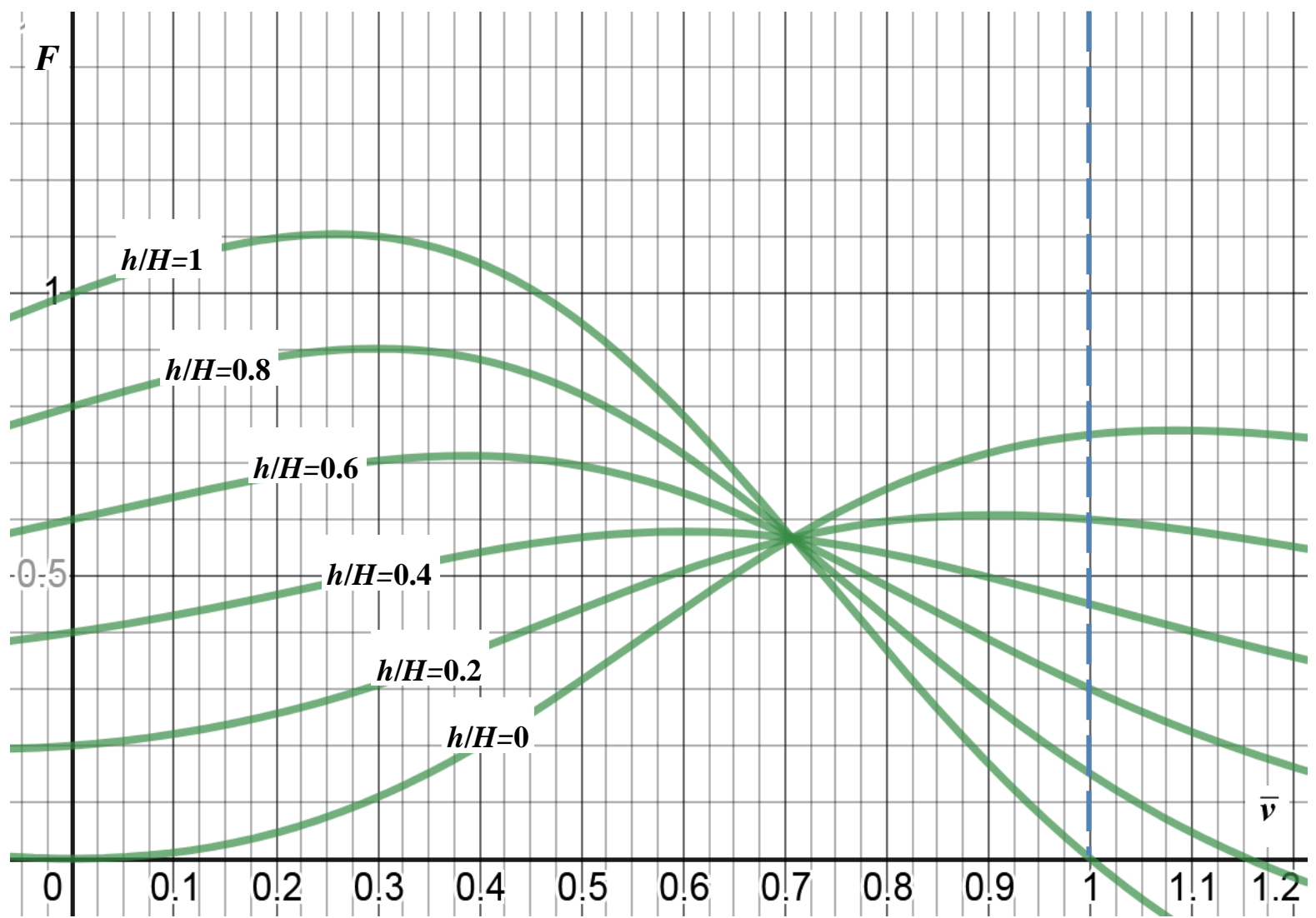

Рис. 3. Зависимость безразмерного перепада давлений $F$ от глубины источника водопритоков $0 \leq h / H \leq 1$ и безразмерной скорости движения воздуха по стволу $0 \leq \bar{v} \leq 1 \bar{v}$

Fig. 3. Dependence of the dimensionless pressure drop $F$ on the depth of the source of water flows $0 \leq h / H \leq 1$ and the dimensionless air velocity along the trunk $0 \leq \bar{v} \leq 1$

\section{СПИСОК ЛИТЕРАТУРЫ}

1. Kazakov B.P., Shalimov A.V., Semin M.A. Stability of natural ventilation mode after main fan stoppage // International Journal of Heat and Mass Transfer. - 2015. - V. 86. - P. 288-293.

2. Шалимов А.В. Устойчивость конвективного движения воздуха в шахтных стволах // Горное эхо. - 2019. - № 1 (74). C. $83-87$.

3. Казаков Б.П., Шалимов А.В. Устойчивость конвективного проветривания рудника после отключения вентилятора // Физико-технические проблемы разработки полезных ископаемых. - 2019. - № 4. - С. 122-130.

4. Шахрай С.Г., Курчин Г.С., Сорокин А.Г. Экспериментальное исследование эффективности естественного проветривания карьера через трубопроводы // Известия высших учебных заведений. Горный журнал. - 2019. - № 1. - С. 127-133.

5. Сологуб О.В Влияние естественной тяги на проветривание Краснополянского тоннеля // Горный информационноаналитический бюллетень. - 2009. - № 1. - С. 32-34.

6. Аэрология горных предприятий / К.3. Ушаков, А.С. Бурчаков, Л.А. Пучков, И.И. Медведев. - М.: Изд-во «Недра», 1987. $421 \mathrm{c}$.

7. Денисенко Е.А. Влияние капежа в вертикальных шахтных стволах на проектирование плановых координат гравитационным способом // Сборник научных трудов Донбасского государственного технического университета. - 2012. - Вып. 38. C. $60-69$.

8. Демочко С.И., Кузнецов А.В., Паршинцев В.П. Неисправности шахтных вентиляторных установок главного проветривания. - М.: Изд-во «Недра», 1990. - 188 с.
9. De Souza E. The mystery of the ventilation raises // Proc. of the 16th North American Mine Ventilation Symposium. - Golden, USA: Colorado School of Mines, 2017. - P. 135-145.

10. Brake D.J. Mine ventilation: a practitioner's manual. - Brisbane, Australia: Mine Ventilation, 2012. - 791 p.

11. Environmental discharge criteria and dispersion estimation for mine ventilation exhaust stacks / W. Harris, A. Kadiayi, K. Macdonald, D. Witow // Proc. of the First International Conference on Underground Mining Technology. - Perth, Australia: Australian Centre for Geomechanics, 2017. - P. 103-113.

12. Viljoen J., Von Glehn F. Investigation of water build-up in vertical upcast shafts through CFD analysis // Proc. of the 11th International Mine Ventilation Congress. - Singapore: Springer, 2019. - P. 1003-1014.

13. Schulz P. Problems of 'water-lock rupture' in a mine ventilation shaft. II. Stability of suspension flow // Archiwum Górnictwa, 1985. - № 30 (2). - P. 235-241.

14. McPherson M.J. An analysis of the resistance and airflow characteristics of mine shafts // Fourth International Mine Ventilation Congress. - Brisbane-Queensland, Australia, 1988. P. 25-33.

15. Semin M., Zaitsev A. On a possible mechanism for the water build-up formation in mine ventilation shafts // Thermal Science and Engineering Progress. - 2020. - V. 20. - P. 107-116.

16. Рогов В.П. Коэффициент сопротивления частиц и капель // Научные труды Дальрыбвтуза. - 2007. - № 19. - С. 95-105.

17. ГОСТ Р 53613-2009. Воздействие природных внешних условий на технические изделия. Общая характеристика. Осадки и ветер. - М.: Стандартинформ, 2011. - 12 с. 
18. Зайцев А.В. Теоретические и технологические основы ресурсосберегающих систем нормализации теплового режима глубоких рудников // Стратегия и процессы освоения георесурсов. - 2018. - Вып. 16. - С. 257-262.

19. Harmathy T.Z. Velocity of large drops and bubbles in media of infinite or restricted extent // AIChE Journal. - 1960. - № 6 (2). P. 281-288.

\section{Информация об авторах}

Шалимов A.B., доктор технических наук, ведущий научный сотрудник отдела аэрологии и теплофизики Горного института УрО РАН.

Казаков Б.П., доктор технических наук, главный научный сотрудник отдела аэрологии и теплофизики Горного института УрО РАН.

Зайцев A.B., доктор технических наук, заведующий сектором математического моделирования и информационных технологий, отдел аэрологии и теплофизики Горного института УрО РАН. 
UDC 622.411

\title{
ANALYTICAL APPROACH TO ESTIMATION OF WATER BUILD-UP EFFECT IN MINE VENTILATION SHAFTS
}

\author{
Andrey V. Shalimov 1 , \\ shalimovav@mail.ru \\ Boris P. Kazakov 1 , \\ aero_kaz@mail.ru \\ Artem V. Zaitsev ${ }^{1}$, \\ aerolog.artem@gmail.com \\ 1 Mining Institute of the Ural Branch of the Russian Academy of Sciences, \\ 78a, Sibirskaya street, Perm, 614007, Russia.
}

The relevance of the study is caused by the need to reduce the additional aerodynamic load on the main fans associated with the occurrence of pressure drop caused by water build-up effect in mine ventilation shafts. This negative effect can lead to significant increase in the air resistance of the mine and to subsequent unstable operation mode of main fan. Avoiding this effect is important not only for energy save mine ventilation, but also for preventing emergency stop of the main fan.

Purpose: finding out the causes and mechanism of the water build-up effect with obtaining qualitative and quantitative estimates of the pressure drop value depending on the air velocity, shaft depth and intensity of the groundwater inflows.

Objects: ventilation shafts.

Methods: analytical modeling of moisture condensation and droplet movement in an ascending air flow in ventilation shaft; comparative analysis of experimental and model data.

Results. The authors have analyzed the experimental data on formation and movement of droplet moisture in the ventilation shafts of various mines. It is noted that the water build-up effect is observed in the range of air velocities from 7 to $12 \mathrm{~m} / \mathrm{s}$, but in some cases the effect is absent even despite the presence of thick fog and abundant condensation of moisture on the surface of the shaft lining and other equipment. Three possible scenarios of the effect are considered: accumulation of a large number of drops of a certain size hanging in the air; drops of large size fall along the entire depth of the shaft with a source of moisture release in its upper part and multidirectional movement of drops depending on their size with a source of moisture release located at arbitrary depth. It is established that the first two models do not allow us to obtain a quantitative estimate of the water build-up effect value due to the uncertainty of the fractional composition of drops, the critical size of which increases the pressure drop to infinity. This drawback was eliminated in the third model by introducing the droplet size distribution function, which made it possible to obtain quantitative results. It is proved that the release of moisture from the air cannot be the cause of the water build-up effect, and the cause is the ground water flows into the shaft with intensity of an order of magnitude higher than moisture condensation. Based on the results of numerical modeling, it is shown that the maximum pressure drop occurs when surface groundwater enters the shaft.

\section{Key words:}

Mine ventilation, moisture condensation, hydrostatic cooling, water flows, pressure loss, aerodynamic drag, natural draft, fractional composition.

The study was carried out with the financial support of the Ministry of Science and Education of the Russian Federation under the agreement on state task no. 075-03-2021-374 of December 29, 2020.

\section{REFERENCES}

1. Kazakov B.P., Shalimov A.V., Semin M.A. Stability of natural ventilation mode after main fan stoppage. International Journal of Heat and Mass Transfer, 2015, vol. 86, pp. 288-293.

2. Shalimov A.V. Ustoychivost konvektivnogo dvizheniya vozdukha $\mathrm{v}$ shakhtnykh stvolakh [Stability of convective air movement in mine shafts]. Mountain echo, 2019, no. 1 (74), pp. 83-87.

3. Kazakov B.P., Shalimov A.V. Stability of convective ventilation of the mine after switching off the fan. Journal of Mining Science, 2019, no. 4, pp. 122-130. In Rus.

4. Shaxray S.G., Kurchin G.S., Sorokin A.G. Experimental study of the effectiveness of natural ventilation of a quarry through pipelines. News of higher educational institutions. Mining journal, 2019, no. 1, pp. 127-133. In Rus.

5. Sologub O.V. Influence of natural draft on airing of the Krasnopolyansky tunnel. Mining informational and analytical bulletin, 2009, no. 1, pp. 32-34. In Rus.

6. Ushakov K.Z., Burchakov A.S., Puchkov L.A., Medvedev I.I Aerologiya gornykh predpriyatiy [Aerology of mining enterprises]. Moscow, Nedra Publ., 1987. 421 p.
7. Denisenko E.A. Influence of drop in vertical mine shafts on the design of planned coordinates by gravity method. Collection of scientific papers of the Donbass state technical University, 2012, Iss. 38, pp. 60-69. In Rus.

8. Demochko S.I., Kuzneczov A.V., Parshincev V.P. Neispravnosti shakhtnykh ventilyatornykh ustanovok glavnogo provetrivaniya. Spravochnoe posobie [Malfunctions of main ventilation shaft fan installations. Reference book]. Moscow, Nedra Publ., 1990. 188 p.

9. De Souza E. The mystery of the ventilation raises. Proceedings of the 16th North American Mine Ventilation Symposium, Golden, USA, Colorado School of Mines, 2017. pp. 135-145.

10. Brake D.J. Mine ventilation: a practitioner's manual. Brisbane, Australia, Mine Ventilation Publ., 2012. 791 p.

11. Harris W., Kadiayi A., Macdonald K., Witow D. Environmental discharge criteria and dispersion estimation for mine ventilation exhaust stacks. Proc. of the First International Conference on Underground Mining Technology. Perth, Australia, Australian Centre for Geomechanics, 2017. pp. 103-113.

12. Viljoen J., Von Glehn F. Investigation of water build-up in vertical upcast shafts through CFD analysis. Proc. of the $11^{\text {th }}$ Interna- 
tional Mine Ventilation Congress. Singapore, Springer Publ., 2019. pp. 1003-1014.

13. Schulz P. Problems of 'water-lock rupture' in a mine ventilation shaft. II. Stability of suspension flow. Archiwum Górnictwa, 1985, no. 30 (2), pp. $235-241$.

14. McPherson M.J. An analysis of the resistance and airflow characteristics of mine shafts. Fourth International Mine Ventilation Congress. Brisbane-Queensland, Australia, 1988, pp. 25-33.

15. Semin M., Zaitsev A. On a possible mechanism for the water build-up formation in mine ventilation shafts. Thermal Science and Engineering Progress, 2020, vol. 20, pp. 107-116.

16. Rogov V.P. Coefficient of resistance of particles and drops. Scientific works of Dalrybvtuz, 2007, no. 19, pp. 95-105. In Rus.

17. GOST R 53613-2009. Vozdeystvie prirodnykh vneshnikh usloviy na tekhnicheskie izdeliya. Obshhaya kharakteristika. Osadki i vet er [Impact of natural external conditions on technical products.
General characteristic. Precipitation and wind]. Moscow, Standartinform Publ., 2011. 12 p.

18. Zaytsev A.V. Teoreticheskie i tekhnologicheskie osnovy resursosberegayushchikh sistem normalizatsii teplovogo rezhima glubokikh rudnikov [Theoretical and technological bases of resource-saving systems for normalization of the thermal regime of deep mines]. Strategy and processes of geo-resource development. Collection of proceedings, 2018, Iss. 16, pp. 257-262.

19. Harmathy T.Z. Velocity of large drops and bubbles in media of infinite or restricted extent. AIChE Journal, 1960, no. 6 (2), pp. 281-288

20. McPherson M.J. Subsurface ventilation and environmental engineering. London, Chapman \& Hall Publ., 2009. 824 p.

Received: 19 April 2021.

\section{Information about the authors}

Andrey V. Shalimov, Dr. Sc., leading researcher, Mining Institute of the Ural Branch of the Russian Academy of Sciences.

Boris P. Kazakov, Dr. Sc., chief researcher, Mining Institute of the Ural Branch of the Russian Academy of Sciences. Artem V. Zaitsev, Dr. Sc., head of the sector, Mining Institute of the Ural Branch of the Russian Academy of Sciences. 